\title{
Psychological Education as the basis for the Training of Taxi Drivers in a Megalopolis
}

\author{
Shipilov Anatoly Ivanovich, Konoshenko Bogdan Aleksandrovich, Gnezdilov Gennady \\ Valentinovich, Klimova Elena Mikhailovna, Kiselev Vadim Vasilievich
}

\begin{abstract}
The relevance of the problem is due to an increase in the number of complaints about the quality of taxi companies, an increase in the number of road accidents involving taxi cars, conflicts in the taxi driver - passenger system, and the lack of a professional taxi driver standard in Russia, despite the extremely dynamic development of this sector and the first place by the mass driver's profession over the past fifteen years, which indicates the need to develop effective psychological programs education whose introduction in training drivers megalopolis taxis could lead to minimize these negative aspects of the current state of the taxi service. In this regard, the purpose of this article is to develop a possible version of such a program, its testing and diagnostics for the effectiveness of the formation in taxi drivers of a megalopolis of methods of social interaction with passengers and the management of a taxi company, reducing the level of conflict tension and general mental stress in the framework of their professional work. The leading research method for this problem is the experimental method, which allows evaluating the effectiveness of the impact of psychological education on the psychological characteristics of taxi drivers, affecting the organization and effectiveness of their professional work. The article presents the author's developed program for the psychological education of megalopolis taxi drivers and the results of its application in practice, which indicate a decrease in the level of perception of the conflict between the taxi driver and the participants in their labor interpersonal interaction, and an increase in the level of knowledge of drivers about the characteristics of problems in professional activities. Article materials may be useful for personnel of taxi services organizations engaged in the selection of taxi drivers and their advanced training.
\end{abstract}

Keywords: taxi drivers, psychological education, training, information model of the conflict, the behavior of the individual in a conflict situation.

\section{INTRODUCTION}

The taxi sector in Russia is currently one of the fastest-growing sectors of the provision of services to the population of the country. Permits for the right to engage in

Revised Version Manuscript Received on 16 September, 2019.

* Correspondence Author

Shipilov Anatoly Ivanovich, Ryazan State University named for S. A.

Yesenin, Svobody Street, 46, Ryazan 390000, Russia. E-mail: anshipilov@yandex.ru

Konoshenko Bogdan Aleksandrovich, Moscow Region State University, Radio Street, 10A, Moscow 105005, Russia.

Gnezdilov Gennady Valentinovich, Moscow Region State University, Radio Street, 10A, Moscow 105005, Russia.

Klimova Elena Mikhailovna, Moscow Region State University, Radio Street, 10A, Moscow 105005, Russia.

Federal public establishment «National Research Institute of the Penal system of the Russian Federation»

Kiselev Vadim Vasilievich, Moscow Region State University, Radio Street, 10A, Moscow 105005, Russia. taxi services in Moscow received more than 100 thousand people, and in general in Russia - more than 700 thousand people. According to the Center for Labor Studies of the Higher School of Economics, in our country over the past fifteen years, the driver's profession has been in the first place by mass - more than 5 million people are drivers (Gimpelson and Vishnevskaya, 2017). In other words, every seventh driver in Russia is a taxi driver.

In modern conditions, the emphasis in the training of taxi drivers in a megalopolis is undergoing significant changes: the formation of a driver's ability to provide services to customers and deliver them to their destination on time and in comfort comes to the fore. In this case, the requirements for the psychological training of taxi drivers in a megalopolis are increasing: its sociability, communication culture, the formation of the need to always be neat, neatly dressed, not to abuse bad habits (in particular, not to smoke at the workplace, etc.).

It is necessary to introduce professional standards to enhance the prestige of the profession, clearly defining what a taxi driver should be (Kruglov, 2016). Large companies have not only local systems for training taxi drivers, but also their standards - from the operator answering a phone call and ending with how the taxi car is delivered. Nowadays, proposals are being made to train taxi drivers in legal literacy, ethics, and foreign languages..

In our opinion, the basis of such training should be the psychological preparation of drivers, especially in conflict management issues, the formation of skills to constructively resolve emerging conflict situations during their work, both with passengers and with the leadership of a taxi company (Konoshenko, 2019).

As part of a system-information analysis of the work of taxi drivers in a megalopolis, the number of indicators was determined:

- the level of knowledge of taxi drivers in a megalopolis about the specifics of their work (interest in the profession of a taxi driver, features of relations with participants in professional activities and conflicts with them, strategies for taxi drivers in conflict situations);

- the perception of a conflict situation by taxi drivers (as limiting the driver's behavior or representing relative freedom in a conflict interaction).

Based on the presented provisions, a scheme was developed to analyze the distortion of drivers' perceptions of conflict with passengers (Table 1). The presented operationalization of indicators is consistent with the analysis scheme of the information model of the conflict interaction of a taxi driver, which forms the basis of an experimental study. Indicators and indicators of the 
information model are recorded using the answers to the

start of the program and after its completion. questionnaire proposed to the study participants before the

Table 1. Analysis scheme of the knowledge level of taxi drivers in a megalopolis about conflict interaction with passengers

\begin{tabular}{|c|c|c|c|}
\hline & Indexes & Criterions & Indicators \\
\hline \multirow[t]{2}{*}{$\begin{array}{l}\text { Taxi driver } \\
\text { knowledge } \\
\text { level: }\end{array}$} & $\begin{array}{l}\text { Relations between } \\
\text { opponents } \\
\text { (definition, functions, } \\
\text { roles) }\end{array}$ & $\begin{array}{l}\text { - definition of their duties; } \\
\text { - determination of the degree of influence } \\
\text { of the situation on professional relations; } \\
\text { - Assessment by drivers of the relationship } \\
\text { between passengers and management; }\end{array}$ & \multirow{2}{*}{$\begin{array}{c}\text { - the meaning of concepts; } \\
\text { - the frequency of use of essential } \\
\text { words; } \\
\text { - description volume (number of } \\
\text { words). }\end{array}$} \\
\hline & \begin{tabular}{|c|} 
Conflict \\
interaction (causes, driver \\
quality behavior in \\
conflict);
\end{tabular} & $\begin{array}{l}\text { - determination of the causes of the } \\
\text { conflict; } \\
\text { - determination of conflict resolution } \\
\text { methods; }\end{array}$ & \\
\hline \multirow{4}{*}{$\begin{array}{l}\text { Perception of } \\
\text { conflict: }\end{array}$} & $\begin{array}{c}\text { Perception of oneself in a } \\
\text { conflict (assessment of } \\
\text { one's "I") }\end{array}$ & $\begin{array}{l}\text { - description of his condition } \\
\text { in conflict } \\
\text { interaction; }\end{array}$ & $\begin{array}{c}\text { a description of yourself and your } \\
\text { behavior in the conflict }\end{array}$ \\
\hline & $\begin{array}{l}\text { Perception } \\
\text { opponent } \\
\text { in conflict } \\
\end{array}$ & $\begin{array}{l}\text { - determination of the hierarchy of } \\
\text { psychological characteristics of the } \\
\text { opponent's personality; }\end{array}$ & conflict opponent description \\
\hline & $\begin{array}{l}\text { Conflict perception } \\
\text { (situation description) }\end{array}$ & - a description of the conflict interaction; & $\begin{array}{c}\text { conflict description: } \\
\text { - meanings in the description; } \\
\text { - frequency of words and } \\
\text { phrases; } \\
\text { - description volume (number of } \\
\text { words). }\end{array}$ \\
\hline & $\begin{array}{l}\text { Perception } \\
\text { action } \\
\text { in conflict }\end{array}$ & $\begin{array}{l}\text { - description of behavior } \\
\text { parties to the conflict } \\
\text { (own and opponent); }\end{array}$ & $\begin{array}{l}\text { description of the actions of } \\
\text { opponents in the conflict: } \\
\text { - meanings in the description; } \\
\text { - frequency of words and } \\
\text { phrases; } \\
\text { - description volume } \\
\text { (number of words). }\end{array}$ \\
\hline
\end{tabular}

\section{MATERIALS AND MethodS}

The main methods of implementation of the developed program of psychological enlightenment are active methods of professional and psychological training: practical classes, role-playing games, development of various emergencies in the professional activities of taxi drivers in megacities, individual psychological counseling. And the current and final control overmastering of the given program using testing, conversations, interrogations, supervision over the decision of taxi drivers of practical problems of the organization of their work in the conditions of a megapolis is very important.

Summarizing the obtained data, we can suggest that adequate training for the driver of a taxi driver in a megalopolis includes several blocks with a total duration of up to 16-20 hours of training (2-3 days of preparation). These areas are:

- legal, technical and technological aspects of labor of a taxi driver in a megalopolis;

- Driving specifics and traffic rules in a megalopolis;

- a corporate culture of the profession and organization;

- The culture, ethics, and psychology of customer service; - the basics of conflict resolution, the psychology of personal safety of the driver;
- the organizational and psychophysiological basis of the work and rest of the taxi driver;

- The history and geography of the city, the sights of the megalopolis;

- knowledge of the Russian language and the basics of English in the work of a taxi driver in a megalopolis.

Using a system-information approach A.Ya. Antsupova and A.I. Shipilova in solving conflict interaction and extending it to the interaction of drivers "horizontally" and "vertically" identified the following elements of the analysis of driver's conflict interaction: information exchange between conflict participants in the framework of professional work, the dynamics of the cognitive processes of opponents of conflict interaction that affect the volume and the objectivity of information models of conflict situations among its participants (Antsupov and Shipilov, 2015).

To increase the validity of the results of the questionnaire, before the start of classes on psychological education and after its completion, a survey was conducted on the method for diagnosing personality behavior in a conflict situation K. Thomas.

The level of distortion in the perception by taxi drivers of a megalopolis of conflict with participants in labor social interaction was analyzed by comparing:

- Descriptions of the above indicators of conflict before the 
implementation of the program of psychological education and after training;

- the completeness of the description by taxi drivers of their ideas about relations with passengers and management (definition, functions, roles) and conflict interaction (reasons, taxi driver behavior in a conflict);

- assessments by drivers of their perceptions of the conflict.

The selection in the experimental group was carried out voluntarily, taking into account the level and frequency of conflict interaction with passengers or management, which was evaluated by the method of diagnosing personality behavior in a conflict situation K. Thomas.

The experimental groups were:

- 1st group - taxi drivers with less than 3 years of work experience $(\mathrm{n}=11)$;

- 2nd group - taxi drivers with work experience of more than 3 years $(n=20)$.

Thus, the total number of subjects in 2 experimental groups was 31 drivers with a work experience of 1.5 to 18 years (29 men and 2 women).

The control groups (total 2 groups, $\mathrm{n}=23$ ) were:

- 1st group - taxi drivers with less than 3 years of work experience $(\mathrm{n}=10)$;

- 2nd group - taxi drivers with work experience of more than 3 years $(n=13)$.

All drivers work in the taxi company "Formula Taxi".

Each experimental group was conducted for 9 hours of classes each lasting 2 hours, the total time of the program is 18 hours of training. In addition to classes on the topics of psychological education with drivers, experimental groups were conducted individual counseling on issues that arise in the classroom. As a result, each of the participants in the experimental groups received an average of 2.3 consultations. During the consultations questions were raised: o strong experience with taxi drivers discussing issues of various breaches of labor discipline with the guide; about the negative evaluation of their activities on the part of the passengers; the complexity of the relationships with other drivers, illegally implementing the functions of the cab and the work carsharing companies.

Classes with each of the experimental groups were held in a separate room, specially equipped to eliminate the impact of interfering with external factors. Within the framework of the psychological education program, the following forms of training sessions were implemented: lectures, practical exercises (case study), panel discussions using slide shows, discussion of video fragments, open discussions of problematic issues, work with stimulating materials of psychodiagnostic techniques, thematic presentations.

The program of psychological education provided for classes taking into account individual work schedules of drivers and was implemented within 3 months with a frequency of approximately one lesson (2 hours) per week. An experimental study was conducted from March to May 2019 during the daytime.

\section{RESULTS}

Analyzing the main results of an experimental study, we determine the most significant of them.

Comparative analysis of indicators by the author's questionnaire aimed at studying the information model of the conflict. The dynamics of responses to the questionnaire to assess the information model of the conflict, allows us to note the following patterns.

1. Description by taxi drivers of a conflict between them and participants in labor social interaction.

The content of the answers and the results of their analysis allow us to note that taxi drivers, after training according to the developed program, have become less focused on identifying external, independent factors, have ceased to consider conflicts as external objects, as if existing independently of them.

Taxi drivers, describing the conflict, are more focused on the assignment of individual characteristics of the conflict, which allows us to note a higher level of awareness of the conflict situation, and, consequently, an idea of the possibility of managing it, as well as an assessment of their involvement, through an assessment of their condition.

Taxi drivers began to monitor their emotional state more subtly, which implies the development of the ability to feel the growth of conflict and tension in relationships, which will allow for the more focused formation of relationships with participants in labor social interaction.

Thus, the training of taxi drivers has resulted in a clearer system of differentiation of the conflict pattern in labor relations, as well as an understanding of the basic role that their relationships play and the importance of their role in such conflicts.

Description of taxi drivers features of their behavior and the behavior of the opponent.

Taxi drivers' answers have more changed towards compromise and cooperation, which suggests the use of more adaptive strategies in resolving conflict situations.

In general, a change in the answers of taxi drivers suggests that they will be able to recognize tensions in a relationship earlier and not bring them to conflict interaction, an expanded variability of behavior in a conflict situation is formed.

The content of the answers and the results of their analysis allow us to note that taxi drivers after training according to the proposed program have become less focused on identifying external, independent factors, have ceased to consider conflicts as external objects, as if existing independently of them.

Description by taxi drivers of their perception of conflict interaction and their idea of how this interaction should be.

The content of the answers allows us to note that the taxi drivers of the megalopolis, after preparing for the program under consideration, have become more focused on identifying personal reasons related to conflicts.

The set of answers allows us to identify a significant change in the image of the conflict among taxi drivers of the megalopolis with its transformation to a deeper understanding of the role of individuals entering into relationships related to professional activities and the allocation of the conflict party's role as managing.

Assessment of the 
effectiveness of the psychological education of taxi drivers in a megalopolis was carried out using $\omega 2$, an unbiased estimate of the strength of the effect. This indicator allows you to evaluate the strength of the factor to determine what proportion of changes in the level of knowledge and perception of conflict situations among taxi drivers participating in the psychological education program is related specifically to the impact of psychological education.

An assessment of the external performance criterion for the professional activity of the EG participants was carried out according to a survey of several taxi company managers. Interviewed 6 leaders of different levels who evaluated the external indicators of the professional activity of drivers who were part of the experimental groups.

The following indicators were noted: the number of conflicts in the professional environment (with employees and managers of the organization); complaints from passengers about taxi drivers; delays and other violations of professional activities. The "experts" involved were assessed from the moment of the beginning of the psychological education program and then during 6 months without mentioning the absolute figures of the mentioned indicators.

Among the selected indicators, "experts" noted the following dependencies:

- the number of conflicts in the professional environment (with employees and managers of the organization) decreased by $14.9 \%$, while the decrease in conflicts with managers is more pronounced;

- complaints from passengers against taxi drivers decreased by $11.6 \%$ (phone calls, SMS to the dispatcher, posting comments on the company's website);

- the decrease in lateness and other professional disruptions is less pronounced $-7.4 \%$, which is more determined by the level of culture and education of drivers.

\section{CONCLUSION}

Thus, the results of an experimental study allow us to draw the following conclusions:

1. The knowledge of taxi drivers about the problems of professional activity and the development of relationships within it expands the range of their perceptions of the complexity of this activity and increases personal responsibility. As a result of these dynamics, interpersonal relationships and conflict interaction become more conscious, which implies understanding the feelings of the other person. In general, this reduces the conflict reactions of taxi drivers and increases the level of awareness of the conflict situation and professional relations "horizontally" and "vertically".

2. The information model of the conflict among taxi drivers in the megalopolis at the first stage of the study is distorted due to the influence of stereotypes of perception of their profession, violation of the process of understanding other people, communication and relationships with various categories of their social environment. After completing the training on the developed program of psychological education for taxi drivers, the description of the conflicts more closely describes the internal feelings, assessing their state and the state of the opponent in the conflict in professional activities. The understanding of personal responsibility for the development of the conflict and even the hidden admission of one's guilt in its manifestation is presented more clearly.

3. A qualitative analysis of open answers and descriptions of conflict situations by taxi drivers showed that psychological education allows you to change the elements of the information model of a labor conflict by changing the perception of opponents of the conflict, more differentiated information about yourself (assessment of one's condition, feelings, goals and orientation in the conflict) and changes the image of the opponent in the conflict (understanding of his internal state, his intentions, goals and possible actions), changing ideas about the conflict as a whole (understanding of conflict as a specific part of the relationship).

\section{RECOMMENDATIONS}

Psychological peculiarities of the work of taxi drivers in a metropolitan city require a radical restructuring of the habitual attitude to the training of taxi drivers, the introduction of a mandatory system of professional training of taxi drivers in the megalopolis. The main directions of such training can be: legal, technical and technological aspects of labor of a taxi driver in a megalopolis; driving features and traffic rules in the megalopolis; corporate culture of profession and organization; customer service culture, ethics and psychology; the basics of conflict management, the psychology of personal safety of a taxi driver; organizational and psychophysiological foundations of the work and rest of a taxi driver; the history and geography of the city, the sights of the megalopolis; knowledge of the Russian language and the basics of English in the work of a taxi driver in a megalopolis. The training program for taxi drivers in megalopolises that we have developed has proven its effectiveness and can be used by personnel services of taxi companies to improve the qualifications of taxi drivers and select the most trained personnel for this type of professional activity.

\section{REFERENCES}

1. Antsupov, A. Ya., \& Shipilov, A. I. (2015). Conflictology: a textbook for universities (5th ed.). St. Petersburg: Piter.

2. Gimpelson, V., \& Vishnevskaya N. (2017). Professions in the Russian labor market: the analytical report of HSE Center for Labor Marke Research. Retrieved March 24, 2019, from http://praktika.direktor.ru/news/2789.

3. Konoshenko, B. A. (2019). Psychological features of labor of taxi drivers in a megalopolis. Moscow: Filin.

4. Kruglov, R. (2016). Taxi drivers are offered to adjust. A word about professional standards Retrieved March 24, 2019, from http://transproff.com/archives/3665/. 